\title{
A method to account for shoot scale clumping in coniferous canopy reflectance models
}

\author{
Sampo Smolander ${ }^{\mathrm{a}, *}$, Pauline Stenberg ${ }^{\mathrm{b}}$ \\ ${ }^{a}$ Division of Biometry, Rolf Nevanlinna Institute, University of Helsinki, P.O. Box 4, FIN-00014 Helsinki, Finland \\ ${ }^{\mathrm{b}}$ Department of Forest Ecology, P.O. Box 27, FIN-00014 University of Helsinki, Helsinki, Finland
}

Received 22 January 2003; received in revised form 3 June 2003; accepted 6 June 2003

\begin{abstract}
The three-dimensional structure of a coniferous shoot gives rise to multiple scattering of light between the needles of the shoot, causing the shoot spectral reflectance to differ from that of a flat leaf. Forest reflectance models based on the radiative transfer equation handle shoot level clumping by correcting the radiation attenuation coefficient with a clumping index. The clumping index causes a reduction in the interception of radiation by the canopy at a fixed leaf area index (LAI). In this study, we show how within-shoot multiple scattering is related to shoot scale clumping and derive a similar, but wavelength dependent, correction to the scattering coefficient. The results provide a method for integrating shoot structure into current radiative transfer equation based forest reflectance models. The method was applied to explore the effect of shoot scale clumping on canopy spectral reflectance using simple model canopies with a homogeneous higher level structure. The clumping of needles into shoots caused a wavelength dependent reduction in canopy reflectance, as compared to that of a leaf canopy with similar interception. This is proposed to be one reason why coniferous and broad-leaved canopies occupy different regions in the spectral space and exhibit different dependency of spectral vegetation indices on LAI.
\end{abstract}

(C) 2003 Elsevier Inc. All rights reserved.

Keywords: Leaf area index; Monte Carlo modelling; Radiative transfer models; Remote sensing; Vegetation canopy model

\section{Introduction}

A well known problem in the radiative transfer theory is how small-scale structures should be handled (Knyazikhin, Martonchik, Myneni, Diner, \& Running, 1998; Ross, 1981; Shabanov, Knyazikhin, Baret, \& Myneni, 2000). In coniferous canopies, the dense clumping of needles in the small region occupied by a shoot causes variation in needle area density at the shoot size scale (i.e. from centimeters to decimeters). It is not feasible to include such small-scale variation into any leaf area density distribution that is to be useful in formulating the radiative transfer problem for threedimensional plant canopies. In other words, the "elementary volume", used in formulating radiative transfer problems, should be small enough that essentially no mutual shading between the elements exists but large enough for statistical laws, such as Beer's law, to apply (Ross, 1981). In coniferous

\footnotetext{
* Corresponding author. Tel.: +358-9-4111-0756; fax: +358-9-19122779.

E-mail address: Sampo.Smolander@Helsinki.Fi (S. Smolander).
}

canopies, there is already substantial mutual shading between the needles of a shoot (Oker-Blom \& Smolander, 1988).

A possible way to overcome this problem is to use the shoot as the basic structural element in radiative transfer models for conifers (Nilson \& Ross, 1997). The canopy structure is then described in terms of the spatial and angular distribution of shoots, and the geometrical and spectral properties of leaves are replaced with those of shoots. This approach, using the annual shoot as the basic structural unit, has long been applied in light interception models (Cescatti, 1998; Nilson, Anniste, Lang, \& Praks, 1999; Oker-Blom \& Kellomäki, 1983; Stenberg, Smolander, \& Kellomäki, 1993) and LAI measurement techniques (Chen, Rich, Gower, Norman, \& Plummer, 1997; Stenberg, 1996). A key parameter entering these models is the shoot silhouette to total area ratio (STAR) (Oker-Blom \& Smolander, 1988), which is conceptually analogous to the $G$-function, or the mean projection of unit foliage area, defined for flat leaves (Nilson, 1971). These models have been designed specifically for the estimation of photosynthesis, and the spectral properties of 
shoots have not been considered important because the scattering of photosynthetically active radiation (PAR) by conifer needles is known to be very small (Daughtry, Ranson, \& Biehl, 1989). Some recent canopy reflectance models (Knyazikhin et al., 1998; Kuusk \& Nilson, 2000; Shabanov et al., 2000) have accounted for the effect of smallscale clumping by modifying the $G$-function but in these approaches the shoot has not been explicitly used as the basic element in evaluating the area scattering phase function of the transport equation. The presence of within-shoot multiple scattering has long been recognized (Gates \& Benedict, 1963; Norman \& Jarvis, 1975), but in order to take it into account in radiative transfer models, it is necessary to derive quantitative relationships between the structure and the scattering properties of a shoot.

In this paper, we present a method by which the effect of needle clumping into shoots can be accounted for in canopy reflectance models. The approach was developed using empirical data on Scots pine (Pinus sylvestris L.) together with a previously developed geometrical model of Scots pine shoots. First, we simulated the scattering phase function of a Scots pine shoot for different wavelengths and for different directions of the incoming beam of photons. Secondly, we estimated the shoot-specific but wavelength independent parameter $p_{\mathrm{sh}}$, corresponding to the probability that a photon scattered from the needle surface of the shoot will interact with the shoot again. The parameter $p_{\mathrm{sh}}$ is conceptually similar to the canopy structural parameter ( $p_{\mathrm{i}}$, probability of interaction) defined by Knyazikhin et al. (1998), which links together canopy absorptance and scattering at any two different wavelengths (Panferov et al., 2001). In thermal engineering, a similar concept is known as view factor or shape factor, giving the proportion of radiation emitted from a body that hits the body again (e.g. Holman, 1986).

We show that the parameter $p_{\text {sh }}$ provides a similar link at the shoot level. That is, knowing $p_{\mathrm{sh}}$ and the scattering coefficient of a needle, the scattering coefficient of a shoot for any given wavelength can be calculated by a simple equation. Third, we derive a theoretical relationship between $p_{\text {sh }}$ and the spherically averaged STAR ( $\left.\overline{\mathrm{STAR}}\right)$, and present empirical verification using material on shoot structure and $\overline{\text { STAR }}$ in Scots pine (Stenberg, Palmroth, Bond, Sprugel, \& Smolander, 2001). Results from the shoot level simulations are used to construct a "shoot-like leaf" with similar $G$-value and scattering properties as the shoot.

Canopies composed of flat leaves, shoots, and "shoot-like leaves" were built, and simulations at the canopy level were performed to compare the spectral reflectance of the canopies for similar values of leaf area index (LAI). We used simple Poisson canopies, where the foliage elements (shoots or leaves) were randomly distributed and spherically oriented. Needle reflectance and transmittance were assumed to be similar to those of leaves, the difference between the canopy reflectances thus being caused solely by shoot structure. The main results from the simulations were that: (i) the clumping of needles into shoots produced a wavelength dependent reduction in canopy reflectance as compared to a leaf canopy with similar LAI, and that (ii) the reflectance behavior of the "real" shoot canopy was well approximated by using "shoot-like leaves", thus providing a means for integrating shoot structure into leaf-based reflectance models.

\section{Materials and methods}

\subsection{Description of shoot structure}

The three-dimensional structure of a coniferous shoot gives rise to multiple scattering within the shoot, causing the scattering from a shoot to differ from that of a flat leaf (Fig. 1). For description of shoot structure, we used a geometrical model for Scots pine shoots, and structural data from a previous investigation (Stenberg et al., 2001). The shoot depicted in Fig. 1A was generated from a model using the following assumptions: (i) needles were of the same size and cylindrical in shape, (ii) needle pairs were evenly positioned along the shoot axis, (iii) the angle between needle and shoot axis was constant, (iv) the orientation of
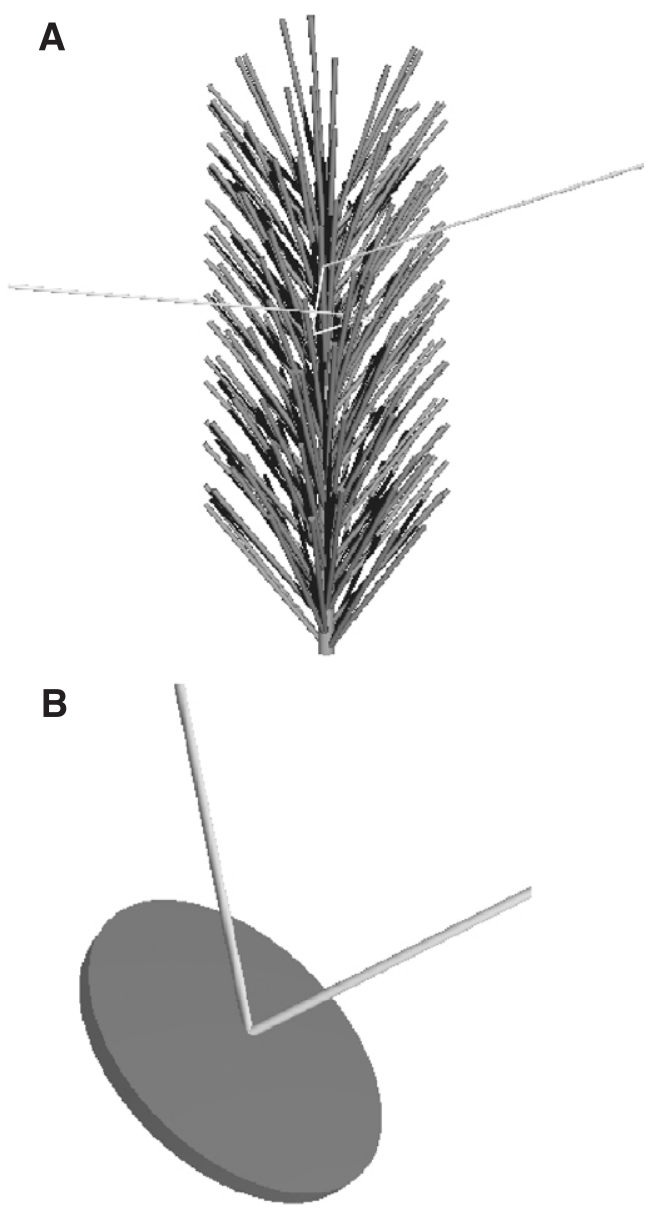

Fig. 1. Presentation of the problem: a photon reflected from a shoot may have interacted with the shoot several times (multiple scattering) (A), whereas with a flat leaf there is only one interaction (B). 
needles around the shoot axis followed a Fibonacci phyllotactic arrangement with a divergence angle of $8 / 13 \times 2 \pi$ between successive needle pairs (Cannell \& Bowler, 1978), and (v) the fascicle angle, which is the opening angle between the two needles in a fascicle, was uniformly distributed between 0 and $2 / 13 \pi$. The structural parameters of the "average" Scots pine shoot chosen for the simulation of scattering phase function are shown in Table 1.

$\overline{\text { STAR }}$ (Table 1) denotes the spherically averaged shoot silhouette to total area ratio, mathematically defined as:

$\overline{\operatorname{STAR}}=\frac{1}{\mathrm{TNA}} \frac{1}{4 \pi} \int_{4 \pi} \operatorname{SSA}(\Omega) \mathrm{d} \Omega$

where TNA denotes the total needle area of the shoot, and $\operatorname{SSA}(\Omega)$ is the shoot silhouette area in direction $\Omega$. Integration over all directions of the sphere is denoted by $4 \pi$. $\overline{\text { STAR }}$ was calculated based on photographically measured SSA in different directions, using the procedure described by Smolander and Stenberg (2001).

\subsection{Simulation of the shoot scattering phase function}

A beam of photons of specific wavelength was fired toward the shoot from different directions, and a ray-tracing procedure was used to follow the path of each photon (this method is called "ray tracing from the light sources" by Foley, van Dam, Feiner, \& Hughes, 1990 to emphasize the fact that the paths of the photons are followed in the direction the photons actually move).

The simulation procedure was as follows. The beam direction was held fixed (entering parallel to the $x$-axis), and the shoot was placed so that its midpoint was in the origin and its axis was along a chosen direction, denoted by $\Omega$. A large number $(N)$ of photons was fired from different $(y, z)$ coordinates chosen randomly from an area $(S)$. This area was defined so as to contain all the shoot silhouette area (SSA) (Fig. 2). From the total number of fired photons, the proportion $\left(N_{\mathrm{i}}^{(\Omega)}\right)$ that hit the shoot (first-order interaction) was followed by ray tracing, whereas the photons that did not hit the shoot were just counted. For a given direction, the fraction of fired photons hitting the shoot $\left(N_{i}^{(\Omega)} / N\right)$ thus corresponds to SSA $/ S$ in the considered direction.

Each photon intercepted by the shoot was followed until it was absorbed, or finally escaped the shoot. By

Table 1

Structural parameters of the model shoot

\begin{tabular}{ll}
\hline Number of needles & 190 \\
Total needle area & $156.3 \mathrm{~cm}^{2}$ \\
Needle length & $2.85 \mathrm{~cm}$ \\
Needle diameter & $0.092 \mathrm{~cm}$ \\
Needle angle (from twig) & $40.5^{\circ}$ \\
Fascicle angle & $0-27.7^{\circ}$ \\
Twig length & $7.7 \mathrm{~cm}$ \\
Twig diameter & $0.3 \mathrm{~cm}$ \\
STAR & 0.133 \\
\hline
\end{tabular}

A

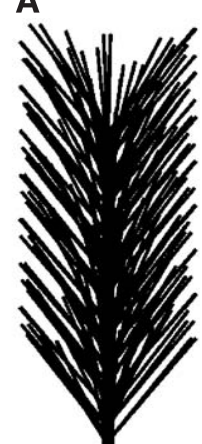

B

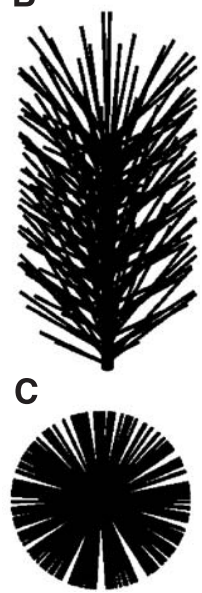

Fig. 2. Silhouettes of the model shoot as seen (A) from side, (B) at $45^{\circ}$ angle, and (C) axially.

intercepted photons, we mean all the photons that originally hit the shoot, regardless of whether they finally were absorbed or scattered. At the points where a photon hit the needle surface, the photon was either absorbed, reflected, or transmitted through the needle. Needle reflectance $\left(\rho_{\mathrm{L}}\right)$ and transmittance $\left(\tau_{\mathrm{L}}\right)$ were assumed to have the same value. Reflection or transmission of a photon hitting a needle occurred with a probability equal to one half of the needle scattering coefficient $\left(\omega_{\mathrm{L}}\right)$ at the specified wavelength, and absorption occurred with probability $1-\omega_{\mathrm{L}}$. The twig was assumed to have similar reflectance as the needles, but transmittance through the twig was set to zero. All surfaces were assumed to reflect as Lambertian surfaces, reradiating the intercepted photons following a cosine distribution around the normal to the surface at the point of reflection. The transmitted part of the radiation was assumed to emerge from a point on the opposite side of the needle, and to follow a cosine distribution around the normal to this opposite surface. (Sometimes in the lower part of a fascicle, the point on the opposite side of the needle was inside the other needle of the fascicle. In this case, the point of emergence was taken to be on the opposite side of that other needle.)

For a Lambertian surface, the fraction of photons reflected into a solid angle $\mathrm{d} \Omega$ around $(\theta, \phi)(=$ polar and azimuth 
angle relative to the needle surface normal at the point of reflection) is given by:

$f(\theta, \phi) \mathrm{d} \Omega=\frac{1}{\pi} \cos \theta \mathrm{d} \Omega=\frac{1}{\pi} \cos \theta \sin \theta \mathrm{d} \theta \mathrm{d} \phi$.

A new direction for a reflected or transmitted photon was generated following this distribution and the ray-tracing procedure was repeated. The final fate of every photon was recorded in terms of: (i) whether it was absorbed or scattered (reflected or transmitted), (ii) its outgoing direction in case of scattering, and (iii) the total number of interactions within the shoot.

The scattering coefficient of a shoot, unlike that of a flat Lambertian surface, varies with the direction of incoming radiation. We define the mean shoot scattering coefficient $\left(\omega_{\mathrm{sh}}\right)$ as the fraction of scattered photons to photons intercepted by the shoot in an isotropic radiation field. Let $\Omega=(\theta$, $\phi)$ denote the orientation of the shoot in relation to beam direction, and $\operatorname{SSA}(\Omega)$ and $\omega_{\mathrm{sh}}(\Omega)$ the silhouette area and scattering coefficient, respectively, of the shoot in the considered orientation. The number of intercepted photons is proportional to $\operatorname{SSA}(\Omega)$, and we have:

$\omega_{\mathrm{sh}}=\frac{1}{4 \pi \overline{\mathrm{SSA}}} \int_{4 \pi} \omega_{\mathrm{sh}}(\Omega) \operatorname{SSA}(\Omega) \mathrm{d} \Omega$,

where $\overline{\text { SSA }}$ denotes the spherically averaged SSA.

Simulation of the scattering phase function of the shoot in an isotropic radiation field was carried out with the procedure described above, with the difference that before a new photon was fired, the orientation of the shoot axis was generated again according to the uniform spherical density function. Interaction between the shoot and a photon from the direction $\Omega$ in relation to the shoot occurred with probability $\operatorname{SSA}(\Omega) / S$, the total fraction of intercepted photons $\left(N_{\mathrm{i}} / N\right)$ being proportional to $\overline{\mathrm{SSA}}$. The shoot scattering coefficient $\left(\omega_{\mathrm{sh}}\right)$ was calculated as the ratio of photons finally escaping the shoot $\left(N_{\mathrm{e}}\right)$ to the total number of photons initially hitting the shoot $\left(N_{\mathrm{i}}\right)$, $\omega_{\mathrm{sh}}=N_{\mathrm{e}} / N_{\mathrm{i}}$. Notice that the shoot scattering coefficient can also be interpreted as the scattering coefficient of a layer of spherically oriented shoots (not shading each other).

\subsection{Estimation of the shoot structural parameter $p_{s h}$}

Simulations of $\omega_{\mathrm{sh}}$ were performed to estimate the shoot structural parameter $p_{\mathrm{sh}}$, expressing the probability that a photon scattered from the needle surface of the shoot will interact within the shoot again. We use a heuristic approach to derive a relation linking the shoot scattering coefficient $\left(\omega_{\mathrm{sh}}\right)$ and absorptance $\left(A_{\mathrm{sh}}=1-\omega_{\mathrm{sh}}\right)$ to the parameter $p_{\mathrm{sh}}$. At every interaction between a photon of specific wavelength $\lambda$ and a needle on the shoot, absorption occurs with probability $1-\omega_{\mathrm{L}}(\lambda)$, where $\omega_{\mathrm{L}}(\lambda)$ is the needle scattering coefficient at the considered wavelength. Otherwise, with probability $\omega_{\mathrm{L}}(\lambda)$, the photon is scattered and may interact within the shoot again (see Fig. 1A). Assuming that the probability by which a scattered photon will interact again (parameter $p_{\mathrm{sh}}$ ) remains constant in successive interactions, shoot absorptance (the eventually absorbed fraction of the photons that initially hit the shoot) is obtained as the sum

$$
\begin{aligned}
A_{\mathrm{sh}}(\lambda)= & {\left[1-\omega_{\mathrm{L}}(\lambda)\right]+\left[1-\omega_{\mathrm{L}}(\lambda)\right] p_{\mathrm{sh}} \omega_{\mathrm{L}}(\lambda) } \\
& +\left[1-\omega_{\mathrm{L}}(\lambda)\right] p_{\mathrm{sh}}^{2} \omega_{\mathrm{L}}(\lambda)^{2}+\ldots=\frac{1-\omega_{\mathrm{L}}(\lambda)}{1-p_{\mathrm{sh}} \omega_{\mathrm{L}}(\lambda)} .
\end{aligned}
$$

Shoot absorptance $\left(A_{\mathrm{sh}}\right)$ normalized by the needle absorptance $\left(1-\omega_{\mathrm{L}}\right)$ equals the average number $(n)$ of interactions between a photon and the shoot. From Eq. (4) follows that

$n=\frac{1}{1-p_{\mathrm{sh}} \omega_{\mathrm{L}}(\lambda)}$

where the denominator $1-p_{\mathrm{sh}} \omega_{\mathrm{L}}(\lambda)$ is the fraction of intercepted photons interacting only once with the shoot. Notice that for $\omega_{\mathrm{L}}=0$, or if $p_{\mathrm{sh}}=0$ (no within-shoot shading), we always have $n=1$ (see Fig. 1).

The shoot scattering coefficient is now obtained (from Eqs. (4) and (5)) as:

$$
\begin{aligned}
\omega_{\mathrm{sh}}(\lambda) & =1-A_{\mathrm{sh}}(\lambda)=\omega_{\mathrm{L}}(\lambda) \frac{1-p_{\mathrm{sh}}}{1-p_{\mathrm{sh}} \omega_{L}(\lambda)} \\
& =\omega_{\mathrm{L}}(\lambda) n\left(1-p_{\mathrm{sh}}\right) .
\end{aligned}
$$

We see that the shoot scattering coefficient normalized by the needle scattering coefficient $\left(\omega_{\mathrm{sh}} / \omega_{\mathrm{L}}\right)$ equals the average number of interactions $(n)$ multiplied by the probability of escape $\left(1-p_{\mathrm{sh}}\right)$. The ratio decreases when the shoot selfshading $\left(p_{\mathrm{sh}}\right)$ increases, and the ratio increases when $\omega_{\mathrm{L}}$ increases. (In case of $p_{\mathrm{sh}}=0$, no within-shoot shading, the scattering coefficients for shoot and needle, $\omega_{\mathrm{sh}}$ and $\omega_{\mathrm{L}}$, are equal.)

\subsection{Relationship between $\overline{S T A R}$ and $p_{s h}$}

If there were no mutual shading between the (assumed convex) needles on a shoot, the spherically averaged shoot silhouette to total area ratio, STAR (Eq. (1)), would be $1 / 4$. (This follows from Cauchy's theorem, stating that the spherically averaged projected area of any convex body equals one fourth of its total surface area; see Lang, 1991). The ratio between STAR and $1 / 4$, i.e. the ratio of spherically projected shoot area to spherically projected needle area, is $4 \overline{\mathrm{STAR}}$. This quantity was defined by Stenberg, Linder, Smolander, and Flower-Ellis (1994) as the shoot shading factor $(\beta)$ (see also Stenberg, 1996), and corresponds to the needle clumping index in shoots $(\kappa)$ used by Nilson et al. (1999). 
We proceed to show that $4 \overline{\mathrm{STAR}}$ can also be interpreted as the mean probability that a photon emitted from a random point on the needle surface of the shoot will not hit another needle of the shoot ("probability of no interaction"). Consider a Lambertian surface of area $A$ emitting radiation at level $E$ per unit area per unit time (see Bell \& Rose, 1981). If $A$ is the surface of a non-self-shadowing (convex) body, an observer from a random direction would see, on average, a silhouette area $A / 4$ at constant radiance $E / \pi$ (since the brightness of a Lambertian surface does not depend on the view angle). Integrating this over all directions $(4 \pi)$ gives a total emitted energy of $A E$ per unit time, as it should. Now, if the object were self-shadowing, its average silhouette area $\overline{A_{\mathrm{S}}}$ would be smaller than $A / 4$. The observer would nevertheless see all of the surface at the same radiance $E / \pi$ (assuming here, that the surface only emits radiation but does not reflect it). Integrating as before, the total emitted energy is $4 \overline{A_{\mathrm{S}}} E$ per unit time. This means, that a proportion of $1-4 \overline{A_{\mathrm{S}}} / A=1-4 \overline{\mathrm{STAR}}$ of the emitted energy does not leave the body, because it hits it again.

We recall that the shoot structural parameter $p_{\mathrm{sh}}$ is the mean probability that a photon reflected from the surface of the shoot will interact with the shoot again. As we assumed Lambertian reflectance, $4 \overline{\mathrm{STAR}}$ can in turn be interpreted as the probability that a reflected photon will escape the shoot. Consequently, $p_{\text {sh }}$ should be closely related to $1-4 \overline{\mathrm{STAR}}$. The only difference between the parameters comes from the spatial averaging: $1-4 \overline{\mathrm{STAR}}$ represents the mean over points on the surface, $p_{\mathrm{sh}}$ is spatially averaged over the points of interaction.

To test the proposed relationship between $p_{\text {sh }}$ and STAR, we estimated $p_{\mathrm{sh}}$ for eight additional Scots pine shoots for which the STAR and parameter values needed in the simulations were available from a previous investigation (Stenberg et al., 2001). The shoots originated from different heights within a tree crown (the same tree from which our model shoot was taken) and represented a wide range of $\overline{\mathrm{STAR}}$ values.

\subsection{Simulation of canopy reflectance}

Canopies with randomly distributed shoots and leaves (Poisson canopies) were generated for different values of LAI. In addition, we constructed a "shoot-like leaf canopy", composed of leaves with the same $G$-value and similar scattering properties as the shoot. Simulations were performed to produce the reflectance of these canopies, assumed to be bounded below by an all-absorbing surface ("black soil"). A beam of photons of a specific wavelength was fired into the canopies from a given direction, and a ray-tracing procedure was applied to follow each photon until it was absorbed, or escaped the canopy.

First, for every fired photon, the length $(l)$ of its path through the canopy before any interaction with leaves/shoots occurred was determined. It was calculated using the prob- ability that a photon, while travelling a distance $x$ in a Poisson canopy, does not interact with leaves,

$P(l \geq x)=\exp \left(-G(\theta) u_{\mathrm{L}} x\right)$

where $u_{\mathrm{L}}$ is the leaf area density and $G(\theta)$ denotes the mean projection of unit leaf or shoot area in the path direction (with zenith angle $\theta$ ).

The leaf area index (LAI) equals leaf area density $\left(u_{\mathrm{L}}\right)$ multiplied by total canopy depth $(D), \mathrm{LAI}=u_{\mathrm{L}} D$. The relationship between canopy interceptance $\left(i_{0}\right.$, the fraction of fired photons interacting with the canopy), corresponding to the probability $1-P(l \geq D / \cos \theta)$, and LAI is given by (cf. Eq. (7))

$i_{0}=1-\exp \left(\frac{-G u_{\mathrm{L}} D}{\cos \theta}\right)=1-\exp \left(\frac{-G \mathrm{LAI}}{\cos \theta}\right)$.

In our model canopies, a spherical orientation of leaves and shoots was assumed. The $G$-value in this case is independent of $\theta$ and equals 0.5 for leaves when leaf area is defined on a half of total surface area basis (Chen \& Black, 1992). For shoots, using half of total needle area as the basis, the $G$-value corresponds to $2 \overline{\mathrm{STAR}}$ (Oker-Blom \& Smolander, 1988). Whenever $l \cos \theta$ was greater than the total depth of the canopy $(D)$, the photon escaped to the soil. Otherwise, the photon interacted with a leaf or a shoot that was assumed to be situated at the sampled depth in the canopy.

When a photon collided with a leaf, the divergence angle $\beta$ (the angle between the propagating direction of the photon before and after collision) and the rotation angle $\psi$ (the angle of rotation for the new direction of propagation, around the axis of the old direction) were sampled according to the probability density function

$f(\beta, \psi)=\frac{2}{3 \pi^{2}}(\sin \beta-\beta \cos \beta)+\frac{2}{3 \pi}(\cos \beta) \frac{\tau_{\mathrm{L}}}{\omega_{\mathrm{L}}}$,

where $\omega_{\mathrm{L}}$ is the leaf reflectance, $\tau_{\mathrm{L}}$ leaf transmittance and where $\beta \in[0, \pi], \psi \in[0,2 \pi]$. This is the scattering phase function for spherically oriented leaves (and is uniform for $\psi)$ as explained in formula (II.6.9) on p. 257 in Ross (1981), but here it is normalized so that its integral over all directions is 1 .

In the shoot canopy, the probability of a photon hitting the shoot from a certain direction should be proportional to the shoot silhouette area on a plane normal to the propagating direction of the photon. This was handled so that, in applying the procedure for spherically oriented shoots, if the photon (fired from a randomly chosen point on the area $S$ of fixed size) did not hit the shoot at the first try, it was fired again from a new point after resampling the shoot orientation. In this way, the probability of interaction with the shoot was proportional to its silhouette area, and the simulated outcome of the photon (absorbed or scattered to a specified direction) 
was representative for spherical shoot orientation. The length of the path of photons scattered from the leaf or the shoot, before new interaction occurred, was sampled again using Eq. (7). Scattered photons escaped the canopy whenever $l$ was greater than the distance out from the canopy in the specified direction.

\subsection{Simulation scheme}

Canopy reflectance was expressed relative to that of an ideal (100\% reflecting) Lambertian surface placed at the top of the canopy. The bidirectional reflectance factor (BRF) is defined as the ratio of radiant flux reflected from a surface area into a particular direction to what would have been reflected in case of an ideal Lambertian surface of the same area (Martonchik, Bruegge, \& Strahler, 2000). A Lambertian surface produces an equal radiance in all directions of the upper hemisphere $(N /(\pi A)$, number of photons per unit solid angle per unit of emitting area normal to the direction of propagation), whereas the total flux (number of photons) into a unit solid angle is proportional to $\cos \theta$ (see Eq. (2)). The number of photons reflected from the canopy to a fixed solid angle $\mathrm{d} \Omega$ around the zenith angle $\theta$ divided by $N \cos \theta \mathrm{d} \Omega / \pi$ gives the BRF. In the simulations, photons entered from a zenith angle of $45^{\circ}$, and BRF was calculated assuming the receiving sensor (with 10 angular radius for $\mathrm{d} \Omega)$ to be in the zenith $\left(\theta=0^{\circ}\right)$.

Canopy BRF of the leaf and shoot canopies was compared for similar values of LAI. Leaf and needle scattering coefficients of 0.1 and 0.9 were chosen to represent wavelengths around the "red edge" in the leaf spectra, characterized by an absorption peak in red and an absorption minimum in near-infrared (NIR). We hereafter refer to the simulated wavelengths as "red" and "NIR".

\section{Results}

\subsection{Shoot scattering phase function}

Fig. 3 shows the directional distribution of photons scattered from the model shoot, for different angles between the directions of the beam and the shoot axis, and also the case for spherically oriented shoots. The photons scattered from the shoot that changed their direction more than $90^{\circ}$ were considered reflected, and photons that changed their direction less than $90^{\circ}$ were considered transmitted. For the NIR wavelength (that is, for $\omega_{\mathrm{L}}=0.9$ ), the shoot scattering coefficient was $\omega_{\text {sh }}=0.81$, with reflectance $\rho_{\text {sh }}=0.47$ and transmittance $\tau_{\mathrm{sh}}=0.34$ (Fig. 3). For the red wavelength $\left(\omega_{\mathrm{L}}=0.1\right)$, the shoot scattering coefficient was $\omega_{\mathrm{sh}}=0.059$, with $\rho_{\mathrm{sh}}=0.034$ and $\tau_{\mathrm{sh}}=0.025$. The scattering phase function of the spherically oriented shoots was closely imitated by the scattering phase function of the shoot-like leaf, for which transmittance $\tau_{\mathrm{L}}$ was $42 \%$ of $\omega_{\mathrm{L}}$ at both wavelengths. Thus, the shoot scattering phase functions had more weight in the backscattering directions than the corresponding leaf scattering phase functions (Fig. 4). (It is to be noted that the shoot transmittance and reflectance, as defined above, are not directly comparable to leaf transmittance and reflectance. With the shoot, photons scattering less than $90^{\circ}$ were always considered transmitted. With spherically oriented leaves, a photon may well scatter less than $90^{\circ}$ also when it is technically reflected, not transmitted.)
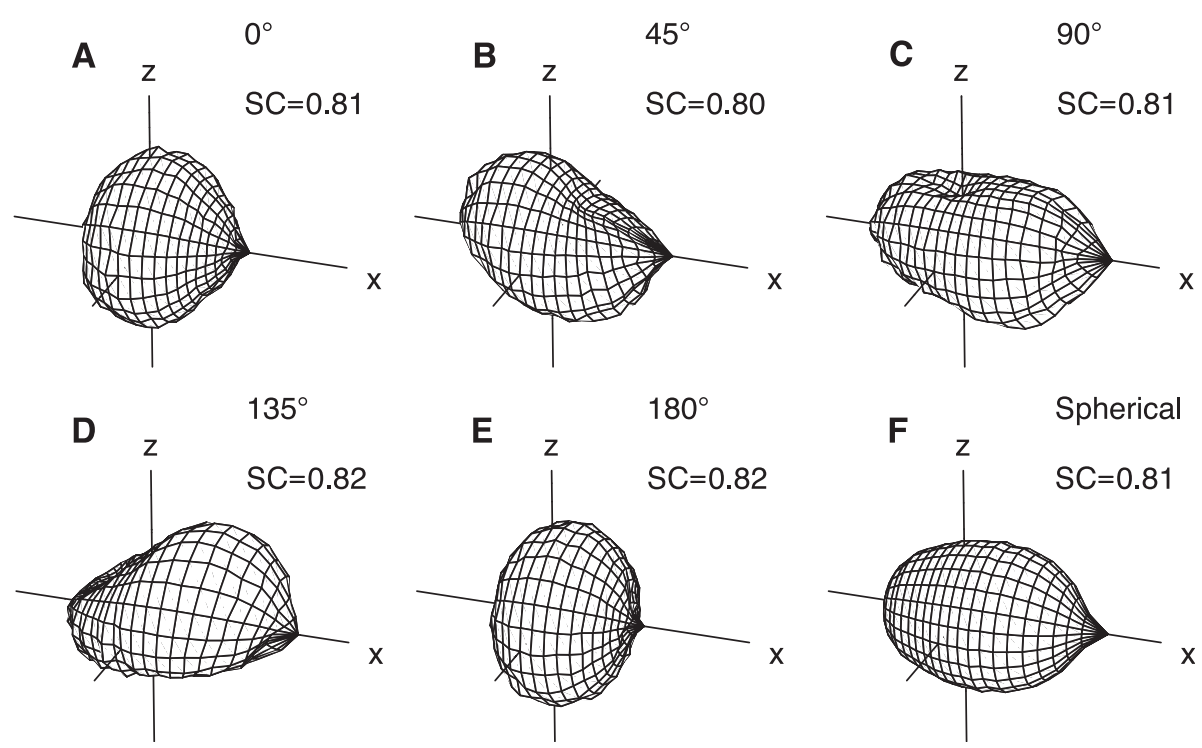

Fig. 3. Shoot scattering coefficient (SC) and the directional distribution of scattered photons for NIR wavelengths (needle $\mathrm{SC}=0.9)$ for different directions of incoming radiation. The beam of radiation enters from the direction of the $x$-axis, and the shoot tip is directed to an angle of (A) $0^{\circ}$ (B) $45^{\circ}$ (C) $90^{\circ}$, (D) $135^{\circ}$, and (E) $180^{\circ}$ opening from the $x$-axis towards the $z$-axis. In (F), the average directional distribution and scattering coefficient for scattering from randomly oriented shoots are presented. 

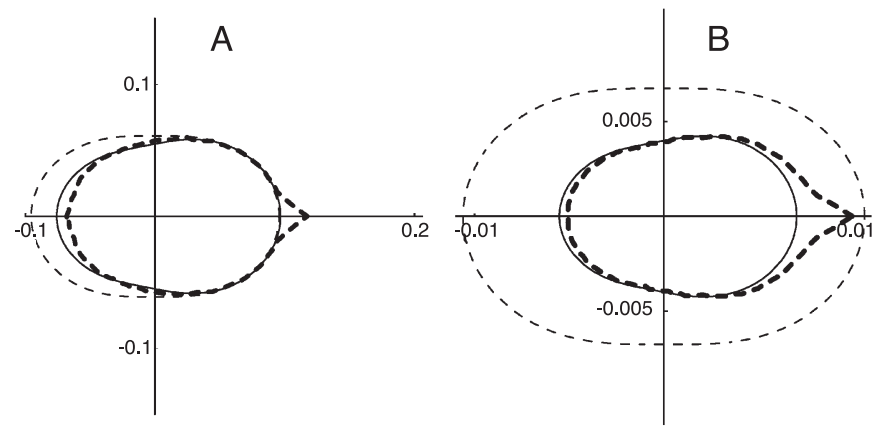

Fig. 4. Cross-sectional views of scattering phase functions for (A) leaf with $\rho_{\mathrm{L}}=0.45, \tau_{\mathrm{L}}=0.45$ (thin dashed line), shoot (thick dashed line) with values $\rho_{\mathrm{L}}=0.45$ and $\tau_{\mathrm{L}}=0.45$ for its needles, and leaf with $\rho_{\mathrm{L}}=0.47$ and $\tau_{\mathrm{L}}=0.34$ (thin line), (B) leaf with $\rho_{\mathrm{L}}=0.05, \tau_{\mathrm{L}}=0.05$ (thin dashed line), shoot (thick dashed line) with values $\rho_{\mathrm{L}}=0.05$ and $\tau_{\mathrm{L}}=0.05$ for its needes, and leaf with $\rho_{\mathrm{L}}=0.034$ and $\tau_{\mathrm{L}}=0.025$ (thin line). The radiation is assumed to come from the direction of positive $x$-axis and to meet the object in origo.

\subsection{Shoot scattering coefficient as a function of the parameter $p_{s h}$}

The shoot scattering coefficient $\omega_{\mathrm{sh}}$ was simulated for a range of needle scattering coefficients $\omega_{\mathrm{L}}=0.1,0.2, \ldots, 1$. The shoot structural parameter $p_{\mathrm{sh}}$ was estimated based on the simulations performed for $\omega_{\mathrm{L}}=1$. It was calculated as the weighted mean of the ratios $n_{j+1} / s_{j}(j=1,2,3, \ldots)$ where $n_{j+1}$ denotes the number of photons interacting at least $j+1$ times with the shoot before being absorbed or scattered out from the shoot, and $s_{j}$ denotes the number of photons scattered at the $j$ th interaction. The ratio gives the fraction of the scattered photons at the $j$ th interaction that hit the shoot again. The ratios were weighted proportional to $s_{j}$. The estimated value of $p_{\mathrm{sh}}$ for the model shoot was 0.474 . Using this value, $\omega_{\mathrm{sh}}$ as a function of $\omega_{\mathrm{L}}$ was then predicted by Eq. (6) (Fig. 5). Good agreement was found between predicted

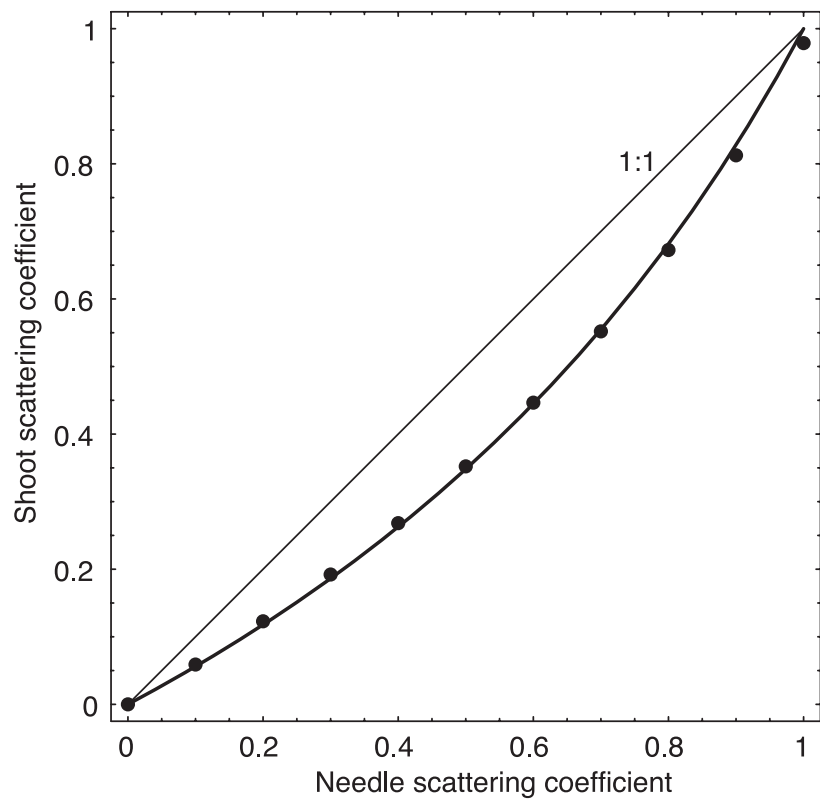

Fig. 5. Predicted (Eq. (5) with $\left.p_{\mathrm{sh}}=0.474\right)$ and simulated shoot scattering coefficient (black dots) for different needle scattering coefficients (wavelengths). and simulated values of $\omega_{\mathrm{sh}}$, despite the fact that there was some variation in the ratios $n_{j+1} / s_{j}$ (i.e. the basic assumption behind the derivation of Eq. (6) did not exactly hold true). Fitting Eq. (6) to the data points in Fig. 5 by the least squares method would have yielded the estimate $p_{\mathrm{sh}}=0.467$, which is negligibly different $(1.5 \%$ difference) from the value estimated directly by simulation as explained above. (The shoot scattering coefficient $\omega_{\text {sh }}$ is 0.98 for the needle scattering coefficient $\omega_{\mathrm{L}}=1$ (Fig. 4) because sometimes the photons hit the twig which had no transmittance.)

\subsection{Correspondence between $\overline{S T A R}$ and $p_{\text {sh }}$}

A close to one to one relationship was found between the simulated $p_{\text {sh }}$ values, and $1-4 \overline{\mathrm{STAR}}$. (Fig. 6). This result is supported by theoretical considerations (Section 2.4), which also explain why an exact correspondence should not be

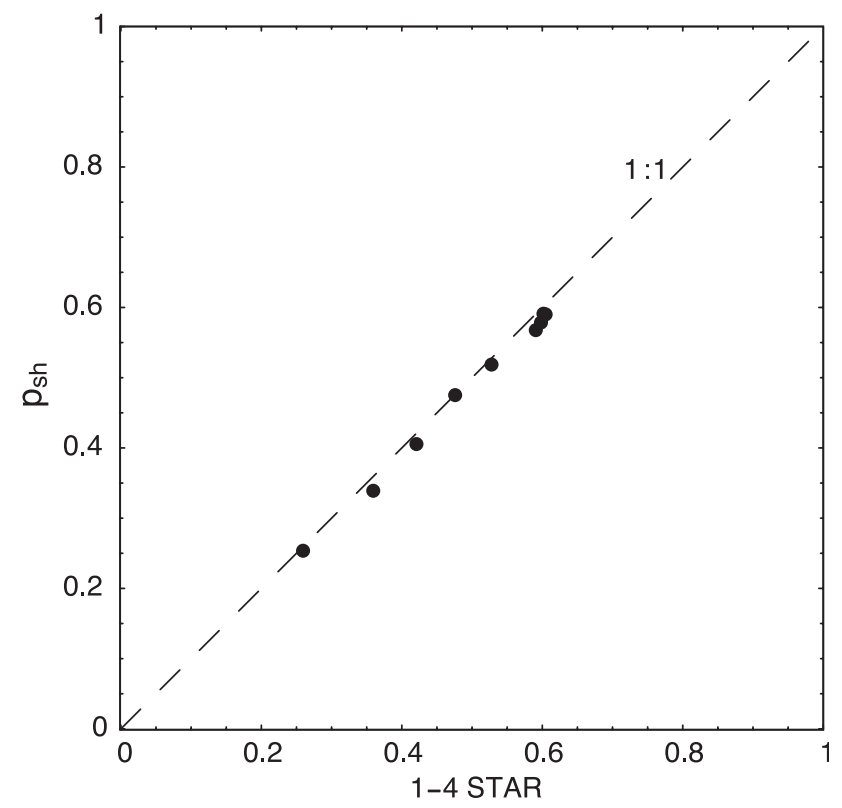

Fig. 6. Relation between $p_{\mathrm{sh}}$ and $1-4 \overline{\mathrm{STAR}}$ for nine Scots pine shoots. Shoot structural data from a previous investigation is used (Stenberg et al., 2001). 
expected. The formulation of the parameter $p_{\mathrm{sh}}$ in Section 2.3 was approximative as it was based on the assumption that the probability of interaction stays constant with successive interactions. In reality, the density of points where scattering occurs varies with the order of interaction, and it is therefore not possible to analytically define the weight on the area over which $p_{\text {sh }}$ is averaged.

It should also be recognized that, in contrast to $\overline{\mathrm{STAR}}, p_{\mathrm{sh}}$ is not just a function of shoot geometry but has some dependency on needle optical properties since they affect the directional distribution of scattered photons (here assumed to be Lambertian). Despite this difficulty in finding a strict definition for $p_{\mathrm{sh}}$ the correspondence between $p_{\mathrm{sh}}$ and $\overline{\text { STAR }}$ was in our case good enough to be useful.
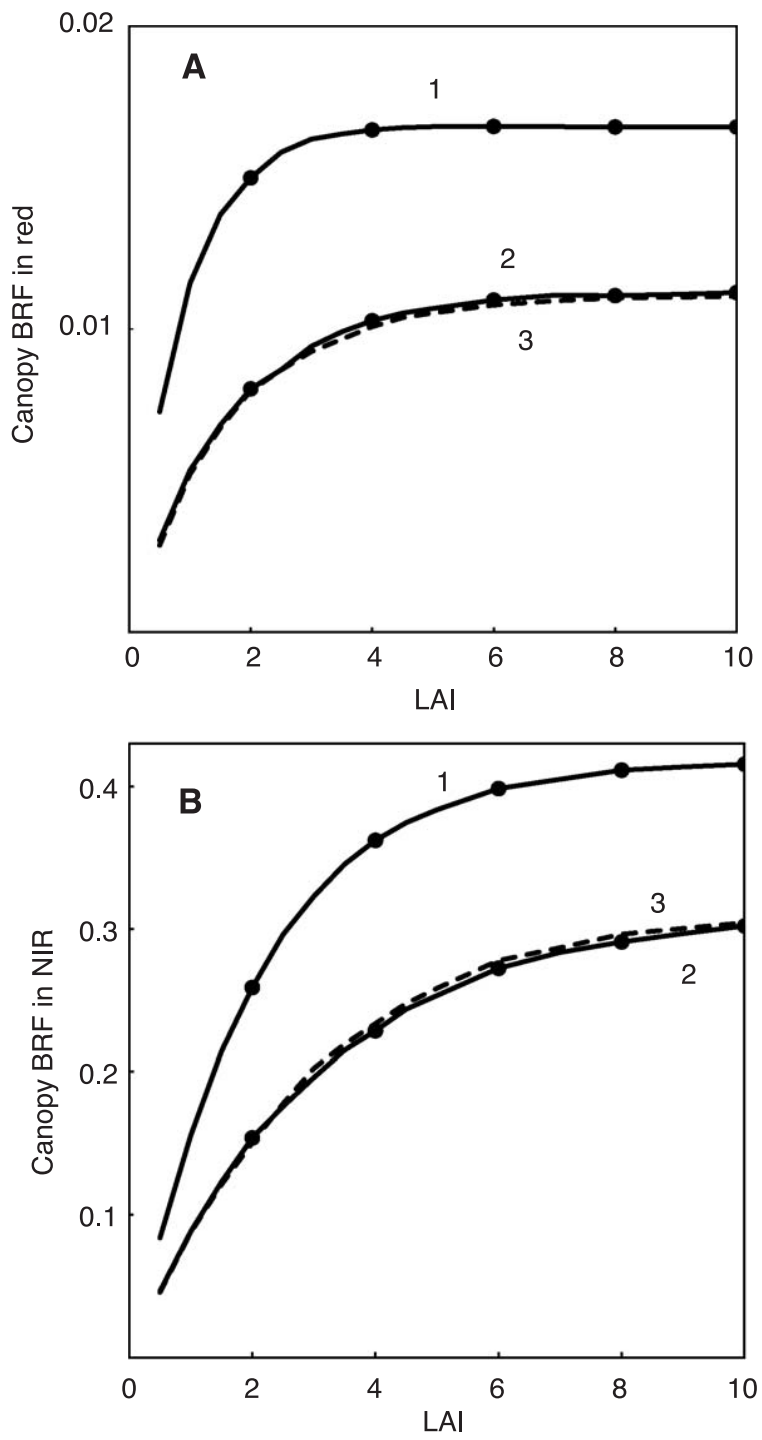

Fig. 7. Canopy bidirectional reflectance factor (BRF) in red and NIR wavelengths as a function of LAI for canopies bounded underneath by black soil. Curve (1) is for leaf canopy, curve (2) for shoot canopy and the dashed curve (3) for "shoot-like leaf" canopy. The black dots denote LAI values of $2,4,6,8$ and 10 . The solar zenith angle is $45^{\circ}$ and the view zenith angle is $0^{\circ}$.
Table 2

Parameters used in the canopy simulations

\begin{tabular}{llllllllll}
\hline Canopy & $G$-value & \multicolumn{2}{l}{ Red } & & & NIR & \\
\cline { 3 - 5 } \cline { 8 - 10 } & & $\omega_{\mathrm{L}}$ & $\rho_{\mathrm{L}}$ & $\tau_{\mathrm{L}}$ & & $\omega_{\mathrm{L}}$ & $\rho_{\mathrm{L}}$ & $\tau_{\mathrm{L}}$ \\
\hline Leaf & 0.5 & 0.1 & 0.05 & 0.05 & & 0.9 & 0.45 & 0.45 \\
Shoot & 0.266 & 0.1 & 0.05 & 0.05 & & 0.9 & 0.45 & 0.45 \\
Shoot-like leaf & 0.266 & 0.059 & 0.034 & 0.025 & & 0.81 & 0.47 & 0.34 \\
\hline
\end{tabular}

\subsection{Canopy simulations}

In Fig. 7, canopy reflectance (BRF) in red and NIR wavelengths for the model canopies (Table 2), bounded underneath by a black surface, are compared. We notice first
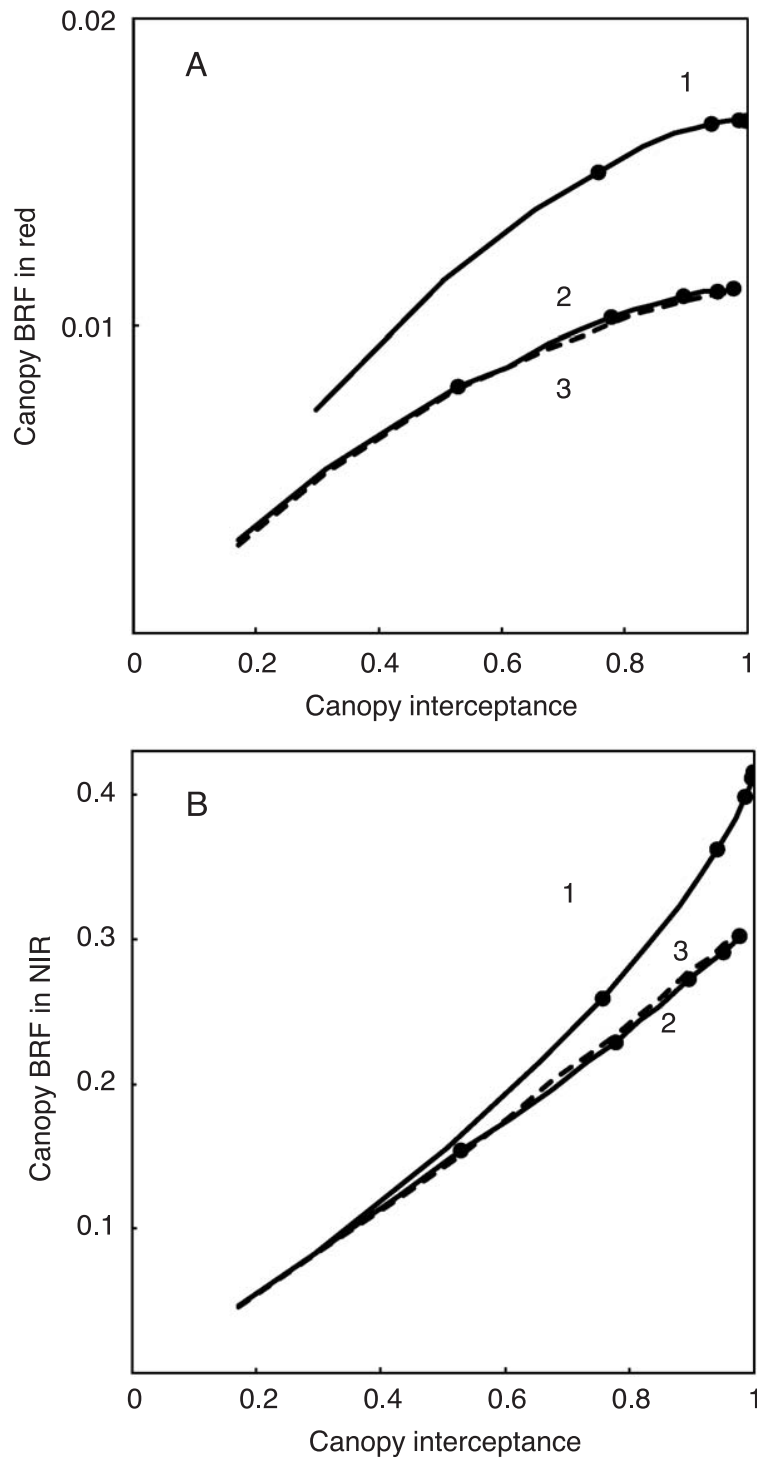

Fig. 8. Canopy bidirectional reflectance factor (BRF) in red and NIR wavelengths as a function of canopy interceptance $\left(i_{0}\right)$ for canopies bounded underneath by black soil. Curve (1) is for leaf canopy, curve (2) for shoot canopy, and the dashed curve (3) for "shoot-like leaf" canopy. The black dots denote LAI values of 2, 4, 6, 8 and 10 to facilitate comparison with Fig. 7. The solar zenith angle is $45^{\circ}$ and the view zenith angle is $0^{\circ}$. 
that the reflectance of the shoot canopy was well approximated by the shoot-like leaf canopy. All curves increased with increasing LAI as they should, since nonreflecting background was assumed in these simulations. At small LAI, canopy reflectance increased more sharply with increasing LAI in the leaf canopy than in the shoot canopy. A reason for this is that the leaf canopy had a higher $G$-value and thus higher canopy interceptance $\left(i_{0}\right)$ at similar LAI (see Eq. (8)). When presented as a function of $i_{0}$ rather than LAI, the reflectance factor of the leaf canopy still remained higher than that of the shoot canopy (Fig. 8). At full canopy cover (represented here by simulations for $\mathrm{LAI}=10$ ) the reflectance factor in red was $1.7 \%$ in the leaf canopy and $1.1 \%$ in the shoot canopy. In NIR, the respective values were $42 \%$ for the leaf canopy and $30 \%$ for the shoot canopy.

\section{Discussion}

For a fixed LAI, the effect of the clumping of foliage in the canopy is to reduce canopy interceptance (fraction of incoming photons interacting with the leaves or needles in the canopy). In radiative transfer models, clumping at larger spatial scales (e.g. grouping of leaves into tree crowns) can conveniently be handled by dividing the canopy volume in non-foliated and foliated parts (tree crowns) (Kuusk \& Nilson, 2000; Nilson \& Peterson, 1991). The spatial distribution of leaf area is commonly described by a probability density function, which allows for variation in leaf area density in different parts of the foliated canopy. However, the scale at which variation can be considered by this approach is limited by the size of the smallest unit (the "elementary volume") for which the statistical description is still reasonable. That is, the (imaginary) elementary volume must be large enough to allow the leaf area density to be defined, and contain a sufficient number of statistically independent foliage elements (see an analogue on p. 8 in Mandelbrot, 1983). To overcome the problem of statistical representation of the distribution of needle area within and between the small regions occupied by coniferous shoots, we have used the shoot as the basic structural unit of the canopy. This was done by deriving the interception, absorption, and scattering properties of a shoot as a function of the shoot structure.

When compared to a single leaf, the effect of mutual shading of needles in a shoot is to decrease the radiation interception efficiency ( $G$-value) and the scattering coefficient of the shoot, and to change the shape of the scattering phase function to weight it more towards the backscattering directions (Fig. 4). We simulated shoot scattering at different wavelengths and derived the wavelength specific mean shoot scattering coefficient $\left(\omega_{\mathrm{sh}}\right)$, representing the scattering coefficient of a layer of spherically oriented shoots (Fig. 3). It was shown that $\omega_{\mathrm{sh}}$ at a specific wavelength could be accurately predicted from the needle scattering coefficient $\omega_{\mathrm{L}}$ at the same wavelength, with the help of a wavelength independent shoot structural parameter, $p_{\text {sh }}$ (Fig. 5). The parameter $p_{\mathrm{sh}}$ - "probability of interaction within the shoot"- depends on the geometrical structure of the shoot. We recall that shoot absorptance $\left(A_{\mathrm{sh}}\right)$ (Eq. (4)) normalized by the needle absorptance $\left(1-\omega_{\mathrm{L}}\right)$ corresponds to the average number $(n)$ of interactions between a photon and the shoot (Eq. (5)), and that the ratio of $\omega_{\text {sh }}$ to $\omega_{\mathrm{L}}$ equals $n$ multiplied by the probability of escape $\left(1-p_{\mathrm{sh}}\right)$. The probability of escape, further, was shown to be closely approximated by the shoot shading factor $\kappa=4 \overline{\mathrm{STAR}}$ (Fig. 6) and thus we have $\omega_{\mathrm{sh}} / \omega_{\mathrm{L}} \approx n \kappa$. The relationship (Eq. (6)) between $\omega_{\text {sh }}$ and $\omega_{\mathrm{L}}$ implies that the larger $p_{\text {sh }}$ (smaller $\kappa$ ), the smaller the ratio $\omega_{\mathrm{sh}} / \omega_{\mathrm{L}}$. At fixed $p_{\mathrm{sh}}$, the ratio $\omega_{\mathrm{sh}} / \omega_{\mathrm{L}}$ increases with $\omega_{\mathrm{L}}$, i.e. the decrease in shoot scattering from mutual shading is relatively less at wavelengths with high needle scattering.

Results from the shoot level simulations were applied to show how the clumping of needles into shoots affects canopy reflectance. The relationship of canopy bidirectional reflectance factor (BRF) with LAI was studied in model canopies built of Poisson-distributed and spherically oriented flat leaves and shoots, respectively (Figs. 7 and 8 ). In the absence of background reflectance, needle clumping decreased the BRF of the shoot canopy as compared to the leaf canopy with similar LAI (Fig. 7) or similar canopy interceptance (Fig. 8). Differences in canopy BRF were relatively larger in the red wavelength (small leaf and needle scattering; $\omega_{\mathrm{L}}=0.1$ ) than in NIR (large leaf and needle scattering; $\omega_{\mathrm{L}}=0.9$ ). In this study, we have only considered the effect of shoot level clumping in canopies with homogeneous higher level structure. Crown mutual shading can also have an important role in the forest reflectance (Gerard \& North, 1997). This kind of higher level clumping would presumably pronounce further the differences between coniferous and broad-leaved forest reflectance. The method introduced in this paper can conveniently be integrated into more realistic forest reflectance models, which take into account the effects of, e.g. background reflectance, crown shape and crown mutual shading.

To parameterize a shoot-like leaf one needs to specify the shoot structural parameter $p_{\mathrm{sh}}$ and the shape of the shoot scattering phase function (Fig. 3). The observed tight relationship between $p_{\mathrm{sh}}$ and $\overline{\mathrm{STAR}}$ is convenient because data on STAR are available for many coniferous species (Cescatti \& Zorer, 2003; Palmroth, Stenberg, Smolander, Voipio, \& Smolander, 2002; Stenberg, Kangas, Smolander, \& Linder, 1999; Stenberg et al., 2001; Stenberg, Smolander, Sprugel, \& Smolander, 1998). The within-shoot hot spot effect was visible as a peak in the backscattering direction in the shoot scattering phase function (Fig. 4) but cannot be described by the bi-Lambertian distribution (Eq. (9)). Although this peak was not included in the shoot-like leaf scattering phase function, the canopy BRF simulations with shoots and shoot-like leaves were in very good agreement (Figs. 7 and 8). However, the simulated canopy BRF was in the zenith direction and not in the backscattering direction where the 
canopy hot spot is seen. The shoot-level backscattering might be important for canopy backscattering.

The shoot scattering coefficient depended on shoot structure $\left(p_{\mathrm{sh}}\right)$ and needle scattering coefficient $\left(\omega_{\mathrm{L}}\right)$ (Fig. 5), but was not sensitive to the ratio of needle transmittance $\left(\tau_{\mathrm{L}}\right)$ to reflectance $\left(\rho_{\mathrm{L}}\right)$ (data not shown). The simplified assumption that $\tau_{\mathrm{L}}$ equalled $\rho_{\mathrm{L}}$ mainly affected the proportions of forward and backward scattering in the shoot scattering phase function (Fig. 4). Changing the ratio of $\tau_{\mathrm{L}}$ to $\rho_{\mathrm{L}}$ (e.g. decreasing $\tau_{\mathrm{L}}$ for visible wavelengths) would change the ratio of shoot forward to backward scattering in the same direction but to a smaller degree. Although the exact shape of the shoot scattering phase function may not have a very large impact on the canopy reflectance, effort should be made to measure realistic spectral values of $\tau_{\mathrm{L}}$ and $\rho_{\mathrm{L}}$ in different species. Similarly, given accurate measurements of needle specular reflectance, this component could be included in the shoot scattering model fairly easily. Finally, to test the model, simulated shoot scattering phase functions should be compared to empirical measurements. To our knowledge such model-based comparison has not been made, although some investigations have involved measurements of the scattering phase function of shoots (Nilson \& Ross, 1997; Ross, Meinander, \& Sulev, 1994).

The main result of this study was the development of an operational method by which the effect of the small-scale clumping of needles into coniferous shoots could be incorporated into forest reflectance models of different types. For this purpose, it was not considered meaningful to construct model canopies with very complex architecture at higher hierarchical levels, so instead we used simple Poissoncanopies with a homogeneous macroscopic structure. Although our simulations for these hypothetical canopies cannot be meaningfully evaluated against real data, results were in qualitative agreement with empirical observations (Häme et al., 2001; Loechel et al., 1997; Nilson et al., 1999; Tian et al., 2000; Zhang, Tian, Myneni, Knyazikhin, \& Woodcock, 2002). That is, it seems that shoot structure can indeed explain large part of the different behavior of coniferous canopy reflectance as compared to broad-leaved canopies. Given relevant data on the geometrical and optical properties of needles and shoots (for the considered species), it would be straightforward to incorporate the approach presented here to radiative transfer models with more realistic description of the macroscopic canopy structure.

$\begin{array}{ll}\text { Notation } \\ \beta & \text { divergence angle } \\ \theta & \text { polar angle } \\ \Omega & \text { a direction (in spherical coordinates) } \\ \omega_{\mathrm{L}} & \text { leaf/needle scattering coefficient } \\ \omega_{\mathrm{sh}} & \text { shoot scattering coefficient } \\ \rho_{\mathrm{L}} & \text { leaf } / \text { needle surface reflectance } \\ \tau_{\mathrm{L}} & \text { leaf } / \text { needle surface transmittance } \\ \phi & \text { azimuth angle } \\ \psi & \text { rotation angle }\end{array}$

$D \quad$ canopy depth

$G \quad G$-function, see Ross (1981)

$i_{0} \quad$ canopy interceptance

LAI leaf area index

$n \quad$ mean number of interactions

$p_{\text {sh }} \quad$ shoot structural parameter

SSA shoot silhouette area

$\overline{\mathrm{SSA}}$ spherical average of SSA

STAR silhouette to total area ratio

$\overline{\text { STAR }}$ spherical average of STAR

$u_{\mathrm{L}} \quad$ leaf area density

\section{Acknowledgements}

The authors are grateful to Prof. Elja Arjas for comments and discussions. The work has been supported by Grants 50178 and 47615 from the Academy of Finland, and by the Graduate School in Computational Biology, Bioinformatics, and Biometry (ComBi).

\section{References}

Bell, C. J., \& Rose, D. A. (1981). Light measurement and the terminology of flow. Plant, Cell and Environment, 4, 89-96.

Cannell, M. G. R., \& Bowler, K. C. (1978). Phyllotactic arrangements of needles on elongating conifer shoots: A computer simulation. Canadian Journal of Forest Research, 8, 138-141.

Cescatti, A. (1998). Modelling the radiative transfer in discontinuous canopies of asymmetric crowns: I. Model structure and algorithms. Ecological Modelling, 101, 263-274.

Cescatti, A., \& Zorer, R. (2003). Structural acclimation and radiation regime of silver fir (Abies alba Mill.) shoots along a light gradient. Plant, Cell and Environment, 26, 429-442.

Chen, J. M., \& Black, T. A. (1992). Defining leaf area index for non-flat leaves. Plant, Cell and Environment, 15, 421-429.

Chen, J. M., Rich, P. M., Gower, S. T., Norman, J. M., \& Plummer, S. (1997). Leaf area index of boreal forests: Theory, techniques and measurements. Journal of Geophysical Research, 102(D24), 29429-29443.

Daughtry, C. S. T., Ranson, K., \& Biehl, L. (1989). A new technique to measure the spectral properties of conifer needles. Remote Sensing of Environment, 27, 81-91.

Foley, J. D., van Dam, A., Feiner, S. K., \& Hughes, J. F. (1990). Computer graphics: Principles and practice (2nd ed.). Reading, MA: AddisonWesley.

Gates, D. M., \& Benedict, C. M. (1963). Convection phenomena from plants in still air. American Journal of Botany, 50, 536-573.

Gerard, F. F., \& North, P. R. J. (1997). Analyzing the effect of structural variability and canopy gaps on forest BRDF using a geometric-optical model. Remote Sensing of Environment, 62, 46-62.

Häme, T., Stenberg, P., Andersson, K., Rauste, Y., Kennedy, P., Folving, S., \& Sarkeala, J. (2001). AVHRR-based forest proportion map of the PanEuropean area. Remote Sensing of Environment, 77, 76-91.

Holman, J. P. (1986). Heat transfer (6th ed.). New York: McGraw-Hill.

Knyazikhin, Y., Martonchik, J. V., Myneni, R. B., Diner, D., \& Running, S. W. (1998). Synergistic algorithm for estimating vegetation canopy leaf area index and fraction of absorbed photosynthetically active radiation from MODIS and MISR data. Journal of Geophysical Research, 103, 32257-32276.

Kuusk, A., \& Nilson, T. (2000). A directional multispectral forest reflectance model. Remote Sensing of Environment, 72, 244-252. 
Lang, A. R. G. (1991). Application of some of Cauchy's theorems to estimation of surface areas of leaves, needles and branches of plants, and light transmittance. Agricultural and Forest Meteorology, 55, 191-212.

Loechel, S. E., Walthall, C. L., de Colstoun, E. B., Chen, J., Markham, B. L., \& Miller, J. (1997). Variability of boreal forest reflectances as measured from a helicopter platform. Journal of Geophysical Research, 102(D24), 29495-29503.

Mandelbrot, B. B. (1983). The fractal geometry of nature. New York: Freeman.

Martonchik, J. V., Bruegge, C. J., \& Strahler, A. L. (2000). A review of reflectance nomenclature used in remote sensing. Remote Sensing Reviews, 19, 9-20.

Nilson, T. (1971). A theoretical analysis of the frequency of gaps in plant stands. Agricultural Meteorology, 8, 25-38.

Nilson, T., Anniste, J., Lang, M., \& Praks, J. (1999). Determination of needle area indices of coniferous forests canopies in the NOPEX region by ground-based optical measurements and satellite images. Agricultural and Forest Meteorology, 98-99, 449-462.

Nilson, T., \& Peterson, U. (1991). A forest canopy reflectance model and a test case. Remote Sensing of Environment, 37, 131-142.

Nilson, T., \& Ross, J. (1997). Modeling radiative transfer trough forest canopies: Implications for canopy photosynthesis and remote sensing. In H. L. Gholz, K. Nakane, \& H. Shimoda (Eds.), The use of remote sensing in the modeling of forest productivity (pp. 23-60). Dordrecht, The Netherlands: Kluwer Academic Publishing.

Norman, J. M., \& Jarvis, P. G. (1975). Photosynthesis in sitka spruce (Picea sitchensis (Bong.) Carr.). Journal of Applied Ecology, 12(3), 839-878.

Oker-Blom, P., \& Kellomäki, S. (1983). Effect of grouping of foliage on the within stand and within-crown light regime: Comparison of random and grouping canopy models. Agricultural Meteorology, 28, 143-155.

Oker-Blom, P., \& Smolander, H. (1988). The ratio of shoot silhouette area to total needle area in Scots pine. Forest Science, 34, 894-906.

Palmroth, S., Stenberg, P., Smolander, S., Voipio, P., \& Smolander, H. (2002). Fertilization has little effect on light-interception efficiency of Picea abies shoots. Tree Physiology, 22, 1185-1192.

Panferov, O., Knyazikhin, Y., Myneni, R. B., Szarzynski, J., Engwald, S., Schnitzler, K. G., \& Gravenhorst, G. (2001). The role of canopy structure in the spectral variation of transmission and absorption of solar radiation in vegetation canopies. IEEE Transactions on Geoscience and Remote Sensing, 39, 241-253.
Ross, J. (1981). The radiation regime and architecture of plant stands. Hague: Kluwer Academic Publishing.

Ross, J., Meinander, O., \& Sulev, M. (1994). Spectral scattering properties of Scots pine shoots. In T. I. Stein (Ed.), Surface and atmospheric remote sensing: Technologies, data analysis and interpretation. International Geoscience and Remote Sensing Symposium IGARSS'94 (pp. 1451-1454). Piscataway: Institute of Electrical and Electronics Engineers.

Shabanov, N. V., Knyazikhin, Y., Baret, F., \& Myneni, R. B. (2000). Stochastic modeling of radiation regime in discontinuous vegetation canopies. Remote Sensing of Environment, 74, 125-144.

Smolander, S., \& Stenberg, P. (2001). A method for estimating light interception by a conifer shoot. Tree Physiology, 21, 797-803.

Stenberg, P. (1996). Correcting LAI-2000 estimates for the clumping of needles in shoots of conifers. Agricultural and Forest Meteorology, 79, $1-8$.

Stenberg, P., Kangas, T., Smolander, H., \& Linder, S. (1999). Shoot structure, canopy openness, and light interception in Norway spruce. Plant, Cell and Environment, 22, 1133-1142.

Stenberg, P., Linder, S., Smolander, H., \& Flower-Ellis, J. (1994). Performance of the LAI-2000 plant canopy analyzer in estimating leaf area index of some Scots pine stands. Tree Physiology, 14, 981-995.

Stenberg, P., Palmroth, S., Bond, B. J., Sprugel, D. G., \& Smolander, H. (2001). Shoot structure and photosynthetic efficiency along the light gradient in a Scots pine canopy. Tree Physiology, 21, 805-814.

Stenberg, P., Smolander, H., \& Kellomäki, S. (1993). Description of crown structure for light interception models: Angular and spatial distribution of shoots in young Scots pine. Studia Forestalia Suecica, 191, 843-850.

Stenberg, P., Smolander, H., Sprugel, D., \& Smolander, S. (1998). Shoot structure, light interception, and distribution of nitrogen in an Abies amabilis canopy. Tree Physiology, 18, 759-767.

Tian, Y., Zhang, Y., Knyazikhin, Y., Myneni, R. B., Glassy, J. M., Dedieu, G., \& Running, S. W. (2000). Prototyping of MODIS LAI and FPAR algorithm with LASUR and LANDSAT data. IEEE Transactions on Geoscience and Remote Sensing, 38, 2387-2401.

Zhang, Y., Tian, Y., Myneni, R. B., Knyazikhin, Y., \& Woodcock, C. E. (2002). Assessing the information content of multiangle satellite data for mapping biomes I: Statistical analysis. Remote Sensing of Environment, $80,418-434$. 